\title{
Highly Transparent, High-Performance IGZO-TFTs Using the Selective Formation of IGZO Source and Drain Electrodes
}

\author{
Hung-Chi $\mathrm{Wu}$ and Chao-Hsin Chien
}

\begin{abstract}
In this letter, high-performance, highly transparent amorphous InGaZnO (IGZO) thin-film transistors (TFTs) with IGZO source/drain electrodes were fabricated. Rapid thermal annealing treatment effectively converted IGZO from a semiconductor into a conductor. Using a patterned $\mathrm{SiO}_{2}$ capping layer, highly transparent IGZO-TFTs with selectively formed IGZO electrodes were fabricated on a glass substrate. The mobility of the fabricated IGZO-TFT was $8.3 \mathrm{~cm}^{2} / \mathrm{V} \mathrm{s}$, ON/OFF ratio was $3.1 \times 10^{6}$, and subthreshold swing was 0.44 V/decade. Thus, the proposed scheme provides a simple and practical method of fabricating high-performance, highly transparent IGZO TFTs.
\end{abstract}

Index Terms-RTA, self-aligned, IGZO, TFT.

\section{INTRODUCTION}

$\mathbf{I}$ $\mathrm{N}-\mathrm{GA}-\mathrm{ZN}-\mathrm{O}$ (IGZO) is an extremely promising candidate for use in amorphous oxide semiconductors because IGZO thin film transistors (TFTs) typically exhibit excellent electrical performance, including high channel mobility, a favorable on/off ratio, a steep subthreshold swing (S.S.), and an acceptable threshold voltage [1]. IGZO is widely used in flexible electronics, TFTs, electronic paper, and sensors, and is positioned to become mainstream in the next generation of TFT technology [2], [3]. However, controlling the composition of oxygen in IGZO is challenging; the ratio of oxygen atom in IGZO and the oxygen bonding state can be drastically influenced by the deposition condition, heat treatment, and other factors [4], [5]. Chasin et al. discovered that changing oxygen content in IGZO by varying oxygen flow during deposition considerably influenced the properties of the contact between electrodes and IGZO. They successfully formed a Schottky contact with metal by using appropriate amounts of IGZO oxygen [6].

Recently, numerous groups have focused on the fabrication of fully transparent, homojunctioned IGZO-TFTs. Ahn et al. demonstrated that plasma treatment can form IGZO into an effective electrode; in addition, $\mathrm{H}_{2}$ plasma treatment increases the carrier concentration in IGZO and effectively

Manuscript received March 23, 2013; revised April 1, 2014; accepted April 8, 2014. Date of publication May 1, 2014; date of current version May 20, 2014. This work was supported by the NSC under Project 101-2628E-009-011-MY3. The review of this letter was arranged by Editor W. S. Wong.

H.-C. Wu is with the Department of Electronics Engineering and Institute of Electronics, National Chiao-Tung University, Hsinchu 300, Taiwan.

C.-H. Chien is with the Department of Electronics Engineering, Institute of Electronics, National Chiao-Tung University, Hsinchu 300, Taiwan, and also with the National Nano Device Laboratories, Hsinchu 300, Taiwan (e-mail: chchien@faculty.nctu.edu.tw).

Color versions of one or more of the figures in this paper are available online at http://ieeexplore.ieee.org.

Digital Object Identifier 10.1109/LED.2014.2317943

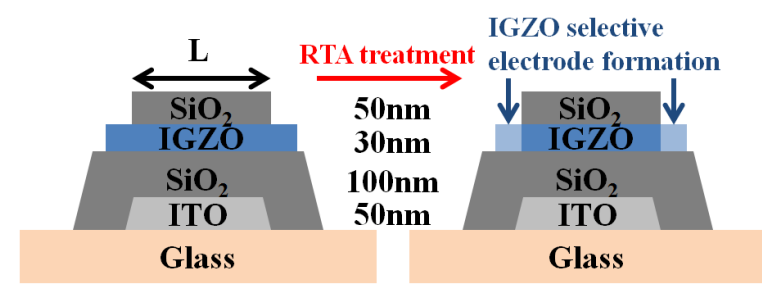

Fig. 1. Schematic structures of IGZO-TFTs in this work.

defines electrode regions [7]. Chen et al. reported that phosphorus implantation enabled IGZO to act as an electrode, demonstrating the possibility of fabricating the IGZO-TFTs by forming self-aligned electrodes [8]. The authors of this study previously demonstrated highly transparent full IGZO-TFTs, in which IGZO was turned into an effective electrode by using rapid thermal annealing (RTA) [9]. However, the previously proposed fabrication process was more complicated compared with the processes used in other studies [7]-[9]; IGZO was required to be deposited twice-once for the electrode, and once for the semiconductor channel region.

In the current study, a novel and simple scheme for fabricating highly transparent, high-performance IGZO-TFTs with selectively formed IGZO electrodes is proposed. Using $\mathrm{SiO}_{2}$ capping on the IGZO channel altered the oxygen bonding states of the uncovered IGZO regions. RTA treatment converted the semiconductor regions into conductor regions, facilitating the formation of highly transparent IGZO TFTs with selectively formed IGZO electrodes.

\section{Device Fabrication}

Fig. 1 shows the schematic IGZO-TFT structure. A glass substrate was used as the starting material. After cleaning the substrate, a 50-nm ITO layer, connected to the pad for probe contacting, was defined and deposited using lithography, and an E-gun provided the gate electrode. $\mathrm{A}-100 \mathrm{~nm} \mathrm{SiO}_{2}$ layer was then formed at $300{ }^{\circ} \mathrm{C}$ by using plasma-enhanced chemical vapor deposition (PECVD). A 200- $\mu \mathrm{m}$ wide, $50-\mu \mathrm{m}$ long channel region was patterned using lithography. Sputtering was then used to deposit $30 \mathrm{~nm}$ of IGZO. The process was previously reported [9]. Next, a $50-\mathrm{nm} \mathrm{SiO}_{2}$ layer was deposited at $150{ }^{\circ} \mathrm{C}$ by using PECVD, and patterned by lithography using the same mask for defining the ITO gate electrode. The channel length was defined based on the length of the $\mathrm{SiO}_{2}$ cap. After fabrication, the devices were treated using RTA at $400{ }^{\circ} \mathrm{C}$ for 1 minute. The electrical characteristics were measured using HP4156 semiconductor parameter 

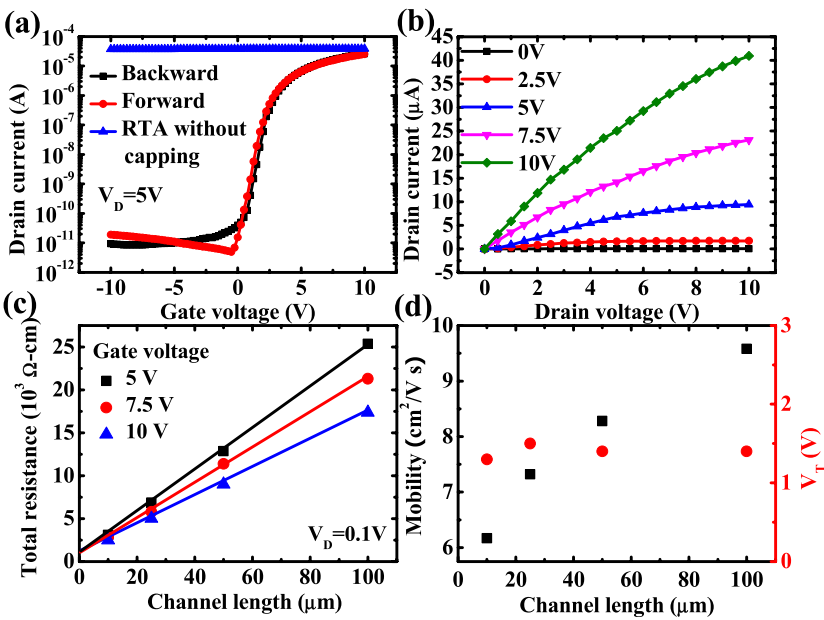

Fig. 2. (a) Transfer and (b) output characteristics of the IGZO-TFTs with $\mathrm{W} / \mathrm{L}=200 / 50 \mu \mathrm{m}$. The blue line in (a) is for the IGZO-TFT subject to the RTA treatment without $\mathrm{SiO}_{2}$ capping. (c) Width-normalized on-state resistance as a function of the channel length at different gate voltages. (d) Mobility and threshold voltage in the linear region as a function of the channel length.

analyzer. The thin film properties were measured using X-ray photoelectron spectroscopy (XPS) and Hall measurement, and the optical transmission measurement of the IGZO film was measured using ultraviolet/visible/near infrared spectroscopy (Hitachi U-4100).

\section{RESUlts AND Discussions}

Fig. 2(a) shows the transfer characteristics of the fabricated IGZO-TFT. The differences between two sweeping directions observed in the off-state indicated numerous trap states in the IGZO channel after RTA [10]. The transfer characteristic of an IGZO-TFT that lacked a $\mathrm{SiO}_{2}$ capping layer yielded only a horizontal line; in other words, no on/off feature was observed. This confirmed the effect of RTA treatment on the properties of IGZO. To determine how RTA treatment affected the interface, an IGZO-TFT involving a Ti electrode, which did not undergo RTA treatment, was also fabricated (not shown). The S.S. was approximately $0.44 \mathrm{~V} /$ decade, which was nearly identical to that of the IGZO-TFT with IGZO electrodes. This result indicated that RTA only influenced the IGZO properties near the surface, and did not substantially affect the interface between the IGZO and $\mathrm{SiO}_{2}$ dielectric layer. Fig. 2(b) shows the corresponding output characteristics; the output behavior was rather normal. Fig. 2(c) shows the channel-width-normalized on-state resistance as a function of the channel length at various gate voltages. The contact resistance extracted using the transfer line method [11] was $713(\Omega-\mathrm{cm})$, which was larger compared with that in previous studies [7] and [8]. Defining the $\mathrm{SiO} 2$ cap by using the same lithography mask as the ITO gate electrode elucidates the upper limit of the contact resistance. In practice, the length of the $\mathrm{SiO} 2$ cap (i.e., channel length) must be defined using a separate mask that exhibits smaller dimensions compared with the gate length, generating reduced contact resistance at the cost of increased gate/drain and gate/source overlap capacitance. Fig. 2(d) exhibits the mobility and threshold voltage in the linear region as a function of the channel length. The threshold voltage did not differ markedly among TFTs demonstrating various channel lengths, implying that RTA treatment did not substantially influence the properties of the
TABLE I

SEVERAL PARAMETERS OF OUR AND Two PREVIOUSLY REPORTED IGZO-TFTS

\begin{tabular}{|c|c|c|c|c|c|}
\hline Method & $\begin{array}{l}V_{\text {th }} \\
(\mathrm{V})\end{array}$ & $\begin{array}{c}\text { Mobility } \\
\left(\mathrm{cm}^{2} / \mathrm{V} \mathbf{s e c}\right)\end{array}$ & $\begin{array}{c}\text { S.S. } \\
\text { (V/decade) }\end{array}$ & $\begin{array}{c}\text { On/Off } \\
\text { ratio }\end{array}$ & $\begin{array}{c}\text { Contact } \\
\text { resistance } \\
(\Omega-\mathrm{cm})\end{array}$ \\
\hline In this work & 1.4 & 8.3 & 0.44 & $3.1 \times 10^{6}$ & 713 \\
\hline Plasma [7] & 0.96 & 7.27 & 0.49 & $1.2 \times 10^{7}$ & 75 \\
\hline Ion implantation[8] & 5.6 & 5 & 0.5 & $6.0 \times 10^{7}$ & 260 \\
\hline
\end{tabular}

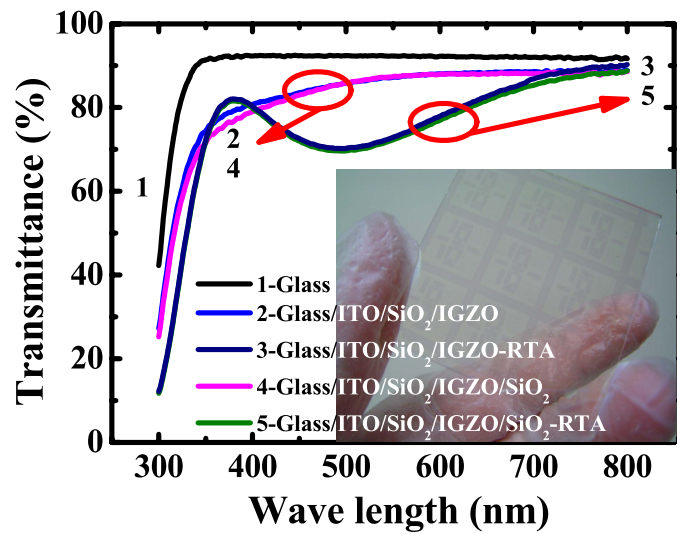

Fig. 3. Transmission spectra of our sample at different stage during IGZO-TFTs fabrication. Inset is the photograph of the fabricated IGZO-TFTs.

IGZO channel. In addition, mobility increased as the channel length increased, indicating that contact resistance limits the effective mobility of the device at short channel lengths; thus, channel length is a critical factor in determining the level of device performance. Table I shows a comparison between the IGZO-TFTs and IGZO electrodes formed using various processes. The electrical performances of the IGZO-TFTs fabricated in this study were comparable to those of electrodes fabricated using plasma and/or ion implantation. Although high contact resistance degraded the electrical performances, the fabricated device exhibited satisfactory device mobility compared with that of devices fabricated in previous studies [7] and [8]. This satisfactory device mobility was attributed to the properties of the IGZO channel rather than the contact resistance. The hydrogen released from the PECVD-deposited $\mathrm{SiO}_{2}$ may diffuse into the IGZO channel and improve device mobility, because of hydrogen can act as a donor in IGZO. A high carrier concentration in the IGZO channel results in increased device mobility [12]. Thus, RTA treatment enables IGZO to act as an electrode, and reducing the contact resistance warrants further investigated. These results elucidate the possibility of fabricating highly transparent, high-performance IGZO-TFTs by using RTA treatment.

Fig. 3 shows the optical transmittance spectra of the sample at various stages of device fabrication without considering reflectance. The samples were high transparency $(70 \%)$ at the wavelengths above $350 \mathrm{~nm}$. However, in the visible region, this drop in transparency was obvious when compared with the reference sample. The degradation may have been caused by the presence of the ITO layer or the effect of thin-film thickness. This result demonstrated that the 

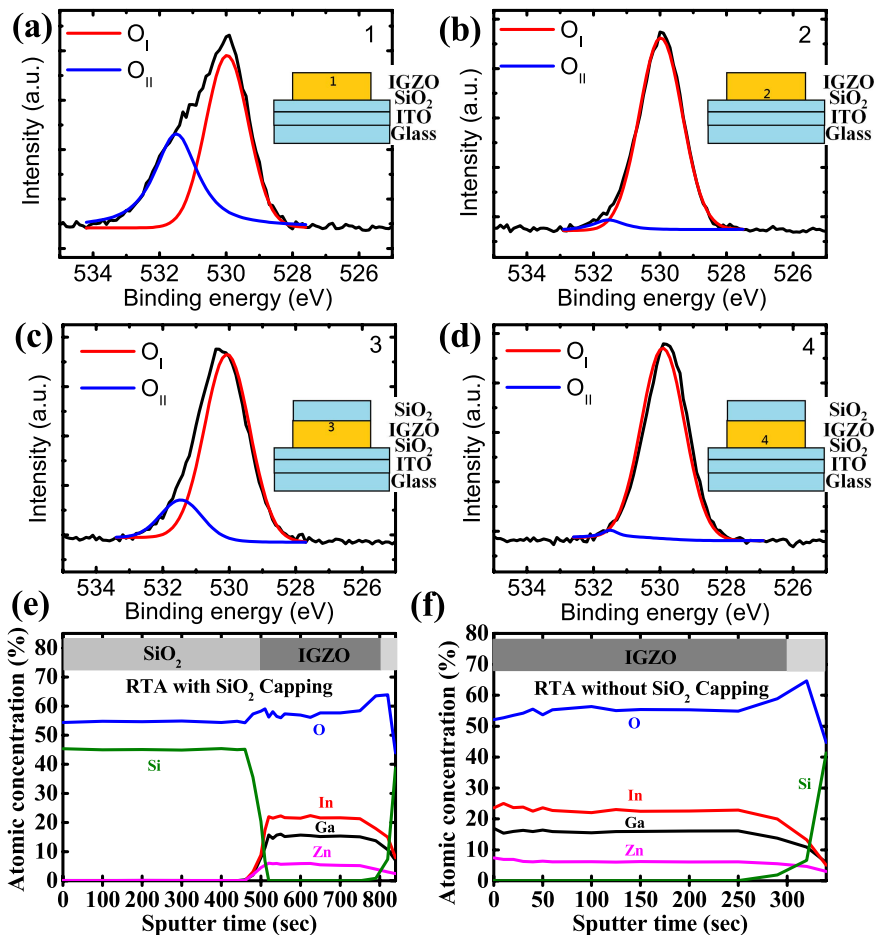

Fig. 4. (a)(b) O 1s XPS narrow scan spectra of the IGZO thin films without $\mathrm{SiO}_{2}$ capping layer during the RTA treatment. (c)(d) The same condition with $\mathrm{SiO}_{2}$ capping layer. The number means the analyzed regions ( 1 and 3 for the surface; 2 and 4 for the bottom). (e)(f) The XPS depth profiles of the IGZO layers with and without $\mathrm{SiO}_{2}$ capping layer after the RTA treatment.

IGZO layer retained a high level of transmittance after RTA treatment. Therefore, high-performance, highly transparent IGZO-TFTs with selectively formed IGZO electrodes were fabricated using the proposed scheme.

Fig. 4 shows the $\mathrm{O}_{1 s}$ XPS narrow scan spectra of IGZO thin film with and without a $\mathrm{SiO}_{2}$ capping layer. All samples were calibrated based on the carbon signal. The IGZO layers with and without $\mathrm{SiO}_{2}$ capping layer yielded considerable differences in $\mathrm{O}_{1 s}$ signals near the surface region The $\mathrm{O}_{1 s}$ peak can be fitted by two nearly Gaussian distributions. $\mathrm{O}_{\mathrm{I}}(529.96 \pm 0.1 \mathrm{eV})$ is related to the $\mathrm{O}_{2}^{-}$ion in the lattice surrounded by the $\mathrm{Zn}, \mathrm{Ga}$ and In atoms in the IGZO compound system. $\mathrm{O}_{\mathrm{II}}(531.55 \pm 0.1 \mathrm{eV})$ is associated with $\mathrm{O}_{2}^{-}$ions located in oxygen-deficient regions within the matrix of IGZO [14] and [15]. The ratio of $\mathrm{O}_{\mathrm{II}}$ state in the surface region was increased in the absence of a $\mathrm{SiO}_{2}$ capping layer after RTA treatment, but no obvious difference in $\mathrm{O}_{\text {II }}$ state ratio was observed in the bottom region in either case. These results further indicated that RTA treatment changes only the properties near the surface region of the IGZO, and maintains the properties of the bottom region. Thus, the IGZO TFT that lacked $\mathrm{SiO}_{2}$ capping did not exhibit an on/off feature after RTA treatment. Figs. 4(e) and (f) show the XPS depth profiles of the IGZO layer with and without a $\mathrm{SiO}_{2}$ capping layer after RTA treatment. A greater reduction in oxygen near the surface region occurred in the IGZO layer that lacked capping, compared with the capped IGZO layer. RTA treatment facilitated oxygen out-diffusion from the IGZO layer, generating oxygen vacancies [9], and resulting in an increased carrier concentration (approximately $10^{20} / \mathrm{cm}^{3}$ ) and Hall mobility of carriers in the IGZO. Therefore, the $\mathrm{SiO}_{2}$ capping layer can be used to stop oxygen out-diffusion during RTA treatment. The effect of RTA treatment is primarily limited to the surface region, and does not affect the entire IGZO region. Furthermore, oxygen vacancies and oxygen bonding states play a critical role in determining the resultant IGZO properties.

\section{Conclusion}

In summary, high-performance and highly transparent IGZO-TFTs with selectively formed IGZO electrodes were developed in this study. The optical transmittance spectra demonstrated that RTA treatment did not considerably degrade the transmittance of IGZO thin film. An XPS analysis demonstrated that the RTA process transformed the oxygen bonding states of the IGZO, causing IGZO to behave as a conductor. In addition, the results indicated that RTA treatment influenced only the surface region of the IGZO layer. The oxygen bonding state in IGZO is essential in determining the electrical performance of IGZO-TFTs. The proposed scheme enables fabricating highly transparent IGZO-TFTs with homojunctioned IGZO electrodes.

\section{REFERENCES}

[1] K. Nomura et al., "Room-temperature fabrication of transparent flexible thin-film transistors using amorphous oxide semiconductors," Nature, vol. 432, pp. 488-492, Nov. 2004.

[2] T. Kamiya, K. Nomura, and H. Hosono, "Present status of amorphous In-Ga-Zn-O thin-film transistors," Sci. Technol. Adv. Mater., vol. 11, no. 4, p. $044305,2010$.

[3] K. Nomura et al., "Thin-film transistor fabricated in single-crystalline transparent oxide semiconductor," Science, vol. 300, pp. 1269-1272, May 2003.

[4] J. S. Park et al., "Review of recent developments in amorphous oxide semiconductor thin-film transistor devices," Thin Solid Films, vol. 520, no. 6, pp. 1679-1693, 2012.

[5] D. Zhang et al., " $\mathrm{In}_{2} \mathrm{O}_{3}$ nanowires as chemical sensors," Appl. Phys. Lett., vol. 82, no. 10, pp. 1613-1615, 2003.

[6] A. Chasin et al., "High-performance a-In-Ga-Zn-O Schottky diode with oxygen-treated metal contacts," Appl. Phys. Lett., vol. 101, no. 11, pp. 113505-1-113505-5, 2012.

[7] B. D. Ahn et al., "Comparison of the effects of Ar and $\mathrm{H}_{2}$ plasmas on the performance of homojunctioned amorphous indium gallium zinc oxide thin film transistors," Appl. Phys. Lett., vol. 93, no. 20, p. 203506, 2008.

[8] R. Chen et al., "Self-aligned indium-gallium-zinc oxide thin-film transistor with phosphorus-doped source/drain regions," IEEE Electron Device Lett., vol. 33, no. 8, pp. 1150-1152, Aug. 2012.

[9] H. C. Wu and C. H. Chien, "High performance InGaZnO thin film transistor with InGaZnO source and drain electrodes," Appl. Phys. Lett., vol. 102, no. 6, pp. 062103-1-062103-4, 2013.

[10] K. Hoshino and J. Wager, Stability of IGZO-Based Thin-Film Transistor: Stability and Temperature-Dependence Assessment of IGZO TFTs. Saarbrücken, Germany: LAP LAMBERT Academic Publishing, 2010.

[11] J. Kanicki and S. Martin, Thin-Film Transistors, C. R. Kagan and P. A. Dekker, Eds. Cleveland, OH, USA: CRC Press, 2003.

[12] J. S. Lee et al., "The influence of the gate dielectrics on threshold voltage instability in amorphous indium-gallium-zinc-oxide thin film transistors," Appl. Phys. Lett., vol. 95, no. 12, pp. 123-502, Sep. 2009.

[13] H.-J. Chang et al., "RF sputtered low-resistivity and high-transmittance indium gallium zinc oxide films for near-UV applications," Electrochem. Solid State Lett., vol. 14, no. 3, pp. H132-H134, 2011.

[14] Y. Wang et al., "Highly transparent solution processed In-Ga-Zn oxide thin films and thin film transistors," Sol-Gel Sci. Technol., vol. 55, no. 3, pp. 322-327, 2010.

[15] B. Kim et al., "Origin of threshold voltage shift by interfacial trap density in amorphous InGaZnO thin film transistor under temperature induced stress," Appl. Phys. Lett., vol. 99, no. 6, p. 062108, 2011. 